\title{
Minimizing the Energy Consumption of a Delta Robot by Exploiting the Natural Dynamics
}

\author{
Rafael Balderas Hill ${ }^{1,2}$, Sébastien Briot ${ }^{2,3}$, Abdelhamid Chriette ${ }^{1,2}$, and \\ Philippe Martinet ${ }^{4}$ \\ 1 Ecole Centrale de Nantes, 44321 Nantes, France \\ 2 Laboratoire des Sciences du Numérique de Nantes (LS2N), UMR CNRS 6004, \\ 44321 Nantes, France \\ 3 Centre National de la Recherche Scientifique (CNRS) \\ 4 Université Côte d'Azur, Inria, 06902 Sophia Antipolis, France
}

\begin{abstract}
It is known that when performing high-speed pick-and-place motions in robot manipulators, an enormous amount of energy is dissipated in order to stop the robot during the braking phase. Typically, this energy is lost as heat in the braking resistances of the motor drivers, leading to increase the energetic losses in the actuation chain. In order to improve the energy efficiency while ensuring accuracy at high-speeds, this paper proposes the use of variable stiffness springs (VSS) in parallel configuration with the motors. This actuation chain is combined with a motion generator which seeks to exploit the robot natural dynamics by adjusting the equilibrium position of the VSS so that the robot is put in near resonance, thus decreasing the energetic losses. Simulations of the suggested approach on a Delta robot are performed and show the drastic reduction of energy consumption.
\end{abstract}

Keywords: variable stiffness springs, pick-and-place, Delta robot

\section{Introduction}

The design trends to operate at high-speeds are changing to the design of energyefficient robots in order to measure as well the robot performance based on the energy consumed by the actuators [1].

The conventional technique to reduce the energy consumption at high speeds consists in designing lightweight robot architectures, thus permitting the use of less powerful motors [2][3]. However, this leads to worsen the robot stiffness, affecting the accuracy of the robot at high speeds. Recently, a novel energyefficient type of actuation, for a SCARA robot in [4], for a five-bar mechanism in [5], and for a Delta robot in [6], proposes the use of constant stiffness springs in parallel to the motors in order to increase the energy efficiency of pick-and-place robots while ensuring accuracy. The main idea of placing the springs in parallel is to distribute the required torque to move the output load between the motor and the spring in parallel. Even if the results for increasing the energy efficiency in [4] [5] [6] are remarkable, the level of adaptation for quasi-periodic motions, i.e. variable travel times and amplitudes, is limited by the spring constant. 
In order to overcome the limitations in terms of adaptability, the authors in [7] for a slow manipulator, and the authors of the present work in [8][9] for highspeed robots, proposed to use variable stiffness springs (VSS) [10] in parallel configuration with the motors. VSS are compliant actuators in which an additional motor is used to vary the stiffness of the springs placed in parallel to the motors that actuate the robot active joints. The work in [7] proposes a controlbased approach by means of a VSS elasticity adaptation law, in which the output stiffness of the VSS can be controlled by means of a nonlinear force/displacement relation for the springs. Even if the results presented in [7] for slow quasi-periodic motions are impressive, the energy required to adjust the stiffness of the VSS is not considered when analyzing the energy savings, which makes difficult to conclude of the real energy gains. In order to solve this lack of energetic analysis, the work in [8] for high-speed robots proposed an energy-based optimal motion generator which minimizes the losses of the full actuation, i.e. robot-plus-VSS, thus considering as well the energy to adjust the VSS. Even if the energy efficiency is increased by $48 \%$, the main issue is that the trajectories are generated by means of polynomials that do not fully exploit the natural dynamics of the system leading to the convergence towards local minima of energy reduction. Hence, the work in [9] presents a strategy to exploit the robot natural motions by adjusting the stiffness of the VSS in parallel so that the robot pseudo-periodic pick-and-place oscillations match the limit cycle of the system. This strategy allows to minimize the input efforts of the parallel robot active joints, and therefore the energy consumed by the actuators by $53 \%$. Nevertheless, the efforts required to adjust the VSS stiffness are not optimized leading to become preponderant when analyzing the energetic losses of the full actuation chain (robot-plus-VSS).

Therefore, this paper improves the results obtained previously in [9], by exploiting the combined motion of the active joints of a Delta robot and the VSS placed in parallel, thus minimizing the robot and VSS energy consumption simultaneously. It is worth emphasizing that for the first time, the use of VSS for performing energy savings is validated on a spatial robot, which was not the case at all in [7][8][9]. This is done thanks to the formulation of a boundary value problem (BVP) in which both, the parallel robot and VSS dynamic equations, are coupled and solved for the desired boundary conditions.

\section{Physical background}

\subsection{Dynamic model of Delta robot with variable stiffness springs in parallel to the motors}

In this section, we will consider the model of a 3-DOF Delta robot with variable stiffness springs in parallel as shown in Fig. 1a. It consists of a base (1) in which three articulated links (2) provide the actuation of the robot in order to move the platform (4), and each articulated arm is connected to a pair of parallel rods (3). Furthermore, three torsional VSS (5) are connected in parallel to the articulated links. This leads to have one of the extremities of the spring connected to a motor, which is coupled at the base, and the other spring extremity connected to the 


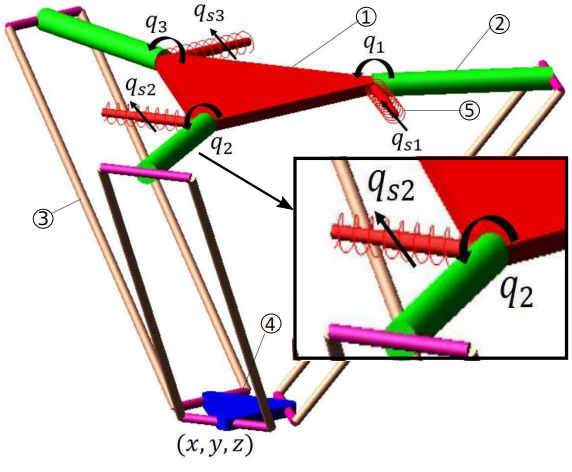

(a) Delta robot and VSS parameterization.

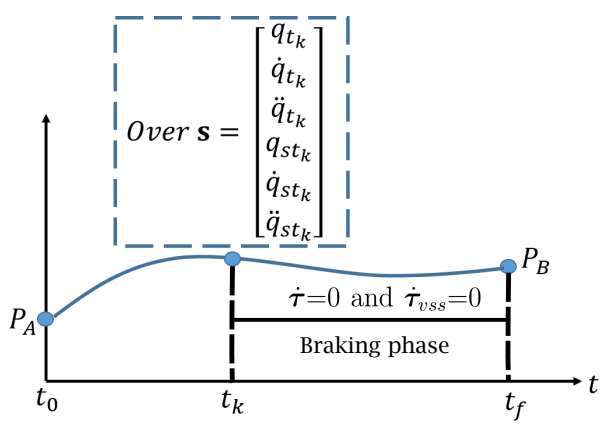

(b) Graphical interpretation motion generator based on BVP.

Fig. 1: On the left, the robot parameterization is defined with three actuated joints $q_{1}, q_{2}$ and $q_{3}$. The variable stiffness torsional springs are located in parallel to the three actuated joints defined by $q_{s 1}, q_{s 2}$ and $q_{s 3}$. On the right, the graphical interpretation of the BVP algorithm for the robot-plus-VSS system is shown.

articulated links (2). The actuation of the three articulated arms is provided by $\mathbf{q}=\left[q_{1}, q_{2}, q_{3}\right]^{T}$ (Fig. 1a). The vector of platform pose is denoted as $\mathbf{x}=[x, y, z]^{T}$. The actuation of the variable stiffness joints is given by $\mathbf{q}_{s}=\left[q_{s 1}, q_{s 2}, q_{s 3}\right]^{T}$. For the dynamic modeling of the Delta robot, the model of a real Delta robot from [11] was used. Skipping all the mathematical derivations and following the Lagrange formalism, the dynamic model of the Delta robot with VSS in parallel to the actuated links can be expressed according to [12], by:

$$
\boldsymbol{\tau}=\left[\begin{array}{c}
\tau_{1} \\
\tau_{2} \\
\tau_{3}
\end{array}\right]=\mathbf{M} \ddot{\mathbf{q}}+\mathbf{c}+\boldsymbol{\tau}_{s}+\mathbf{f}_{a}, \quad \boldsymbol{\tau}_{v s s}=\left[\begin{array}{c}
\tau_{v s s_{1}} \\
\tau_{v s s_{2}} \\
\tau_{v s s_{3}}
\end{array}\right]=\mathbf{J}_{s} \ddot{\mathbf{q}}_{s}-\boldsymbol{\tau}_{s}+\mathbf{f}_{s}
$$

where $\boldsymbol{\tau}$ and $\boldsymbol{\tau}_{v s s}$ represent respectively the vector of robot input torques and the vector of input torques associated to the VSS dynamics. $\mathbf{M}$ is the positive matrix of inertia, $\mathbf{c}$ is the vector of Coriolis, centrifugal and gravitational effects, $\mathbf{f}_{a}$ is the vector of active joint friction terms, $\mathbf{J}_{s}=\operatorname{diag}\left(J_{1}, J_{2}, J_{3}\right)$ represent the inertias of the couplings between the motors and the VSS, and $\mathbf{f}_{s}$ is the vector grouping the variable stiffness joint friction terms. Finally, $\boldsymbol{\tau}_{s}=\mathbf{K}\left(\mathbf{q}-\mathbf{q}_{s}\right)$ is the torque vector associated to the force/displacement relation due to the VSS coupled in parallel to the robot with $\mathbf{K}=\operatorname{diag}\left(k_{1}, k_{2}, k_{3}\right)$ being the spring constants.

\subsection{Energy consumption modeling}

In order to analyze the energetic losses from the motors actuating the Delta robot during the high-speed pick-and-place tasks, we consider the energetic model presented in [8], by the following expression:

$$
\int_{0}^{t_{f}} P_{\text {losses }} d t=\int_{0}^{t_{f}}\left(P_{\text {motor }}+P_{\text {brake }}+P_{\text {damp }}+P_{\text {cond }}+P_{\text {switch }}+P_{\text {rectifier }}\right) d t
$$


where $P_{\text {motor }}$ and $P_{\text {brake }}$ group the resistive losses (from motor and braking phase), $P_{\text {damp }}$ represents the mechanical losses due to mechanical coupling, $P_{\text {cond }}$ groups the conduction losses, $P_{\text {switch }}$ corresponds to the switching losses, and $P_{\text {rectifier }}$ represents the losses due to rectification, $t_{f}$ is the final time. The mathematical expressions describing each power loss can be found in [8].

\section{Exploiting robot natural motions for reducing the energy consumption}

According to [8], in which the model of losses (2) has been analyzed for highspeed pick-and-place tasks, most of the energetic losses are accumulated during the braking phase, i.e. when the motor operation switch to generator mode and the electric currents circulate towards the braking resistors of the motor drivers.

For systems that are required to oscillate at a constant frequency, it is possible to find a unique set of spring constants grouped in matrix $\mathbf{K}$, such that the system remains with a periodic exchange of kinetic and spring potential energy. However, for pick-and-place robots, such as the Delta is not the same as the motions are defined by pseudo-periodic sequences. That is why, by tuning online the VSS we seek to put the robot near a resonance mode for pseudo-periodic motions. In order to do that, based on the dynamic equations in (1), to exploit the robot natural dynamics for the full actuation chain, i.e. the robot-plus-VSS, it is necessary to find a combined motion of the spring and parallel robot coordinates. This is due to the fact that the force/displacement relation $\boldsymbol{\tau}_{s}$, associated to the VSS optimal stiffness and which couples the dynamic expressions (1), is dependent on $\mathbf{q}_{s}$ and $\mathbf{q}$, which are controllable variables. Additionally, since during an energy-efficient pick-and-place operation, there is no restriction for the robot on how to go from the initial to the final positions, except external environment or singularity loci, we only need to ensure accuracy in the boundaries. That is why, here we propose the formulation of a BVP in which by integrating the dynamic equations $\left(\boldsymbol{\tau}, \boldsymbol{\tau}_{v s s}\right)$ for the desired boundary constraints (pick or place states), we calculate an optimal trajectory which satisfies the boundary constraints. This will ensure that the robot behaves as an unforced system, i.e. with minimum input torques, during the braking phase in which the energetic losses increase due to the energy dissipated on the braking resistances.

Typically, in order to parameterize a desired pick-and-place task for a parallel robot, the boundary conditions for positions, velocities and accelerations must be specified. Thus, since we seek to apply the BVP formulation to constrain up to the accelerations of the pick-and-place points, it is required to have higherorder dynamic equations. This is due to the fact that the order of the system in (1) allows to constrain up the velocities of the pick-and-place task. Therefore, we timed differentiate the expressions in (1) to obtain the jerk equations as follows:

$$
\dot{\boldsymbol{\tau}}=\mathbf{M} \ddot{\mathbf{q}}+\dot{\mathbf{M}} \ddot{\mathbf{q}}+\dot{\mathbf{c}}+\mathbf{K}\left(\dot{\mathbf{q}}-\dot{\mathbf{q}}_{s}\right)+\dot{\mathbf{f}}_{a}, \quad \dot{\boldsymbol{\tau}}_{v s s}=\mathbf{J}_{s} \dddot{\mathbf{q}}_{s}-\mathbf{K}\left(\dot{\mathbf{q}}-\dot{\mathbf{q}}_{s}\right)+\dot{\mathbf{f}}_{s}
$$

where the sign functions from the active joint friction terms in $\mathbf{f}_{a}$ and $\mathbf{f}_{s}$, in order to obtain $\dot{\mathbf{f}}_{a}$ and $\dot{\mathbf{f}}_{s}$, respectively, are approximated to be time differentiable. Then, the BVP formulation applied to find the combined spring and 


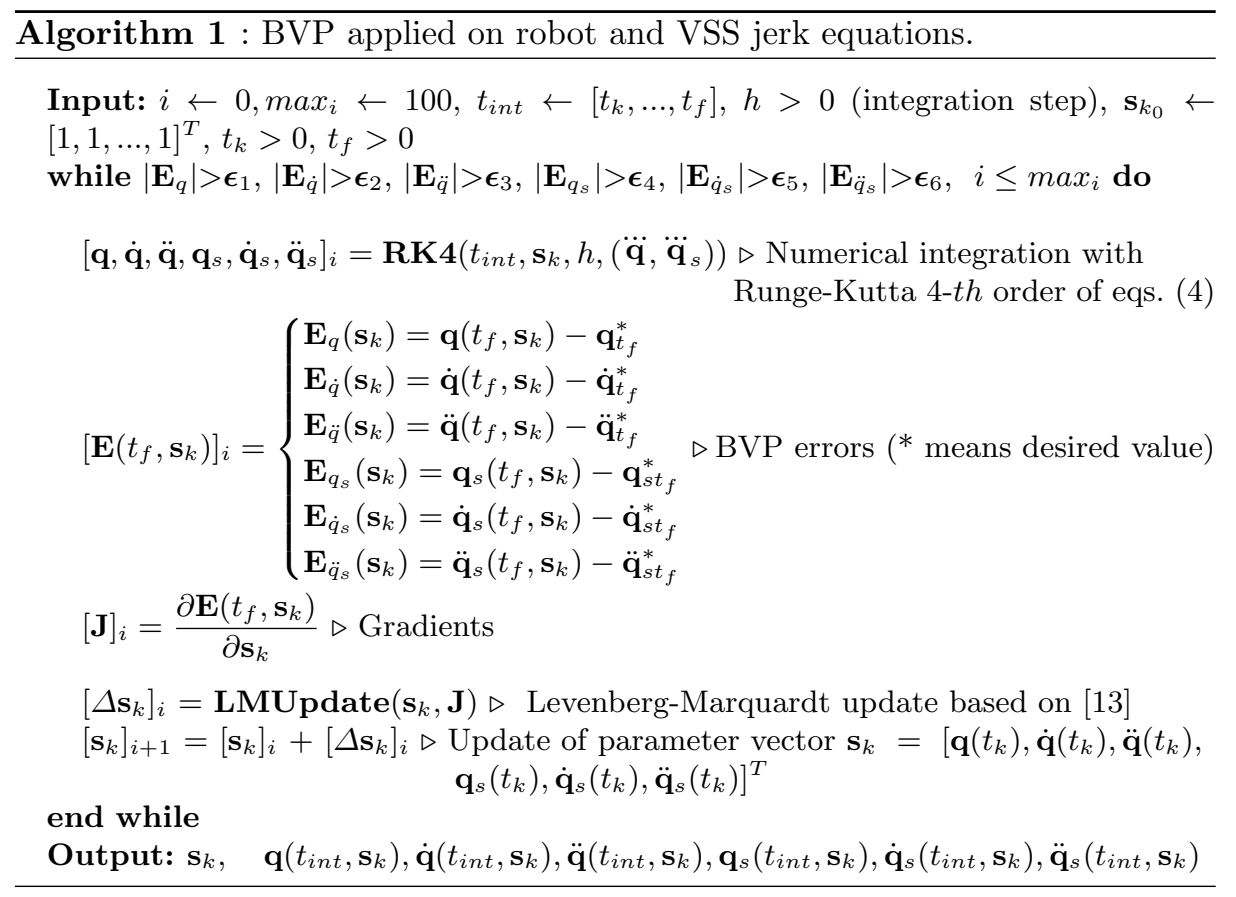

robot coordinates $\mathbf{q}_{s}$ and $\mathbf{q}$, respectively, seeks to solve the coupled robot-plusVSS system (3) for $\dot{\boldsymbol{\tau}}=0$ and $\dot{\boldsymbol{\tau}}_{v s s}=0$, i.e.:

$$
\dddot{\mathbf{q}}=-\mathbf{M}^{-1}\left(\dot{\mathbf{M}} \ddot{\mathbf{q}}+\dot{\mathbf{c}}+\mathbf{K}\left(\dot{\mathbf{q}}-\dot{\mathbf{q}}_{s}\right)+\dot{\mathbf{f}}_{a}\right), \quad \dddot{\mathbf{q}}_{s}=\mathbf{J}_{s}^{-1}\left(\mathbf{K}\left(\dot{\mathbf{q}}-\dot{\mathbf{q}}_{s}\right)-\dot{\mathbf{f}}_{s}\right)
$$

with the boundary conditions defined as the desired positions, velocities and accelerations at the braking phase by $\mathbf{q}\left(t_{f}\right)=\mathbf{q}_{t_{f}}^{*}, \dot{\mathbf{q}}\left(t_{f}\right)=\dot{\mathbf{q}}_{t_{f}}^{*}, \ddot{\mathbf{q}}\left(t_{f}\right)=\ddot{\mathbf{q}}_{t_{f}}^{*}$, and the desired final VSS positions, velocities and accelerations by $\mathbf{q}_{s}\left(t_{f}\right)=\mathbf{q}_{s t_{f}}^{*}$, $\dot{\mathbf{q}}_{s}\left(t_{f}\right)=\dot{\mathbf{q}}_{s t_{f}}^{*}$, and $\ddot{\mathbf{q}}_{s}\left(t_{f}\right)=\ddot{\mathbf{q}}_{s t_{f}}^{*}$ by formulating the nonlinear BVP defined in Algorithm 1. It should be noted that from the aforementioned algorithm, to compute the BVP error vectors grouped in $\mathbf{E}\left(t_{f}, \mathbf{s}_{k}\right)$, it is necessary for $\mathbf{M}$ to be numerically invertible, i.e. out of Type 2 singularity [14]. Thus, in the integration step, an inversion-checking condition representing the proximity to singularity is added to verify the invertibility of the decoupling matrix $\mathbf{M}$.

The different elements of the pseudo-code provided in Algorithm 1 are defined as follows: $\max _{i}$ is the maximum number of iterations $i, \boldsymbol{\epsilon}_{1 \ldots 6}$ represent the BVP errors thresholds, $\ddot{\mathbf{q}}, \dot{\mathbf{q}}, \mathbf{q}$ and $\ddot{\mathbf{q}}_{s}, \dot{\mathbf{q}}_{s}, \mathbf{q}_{s}$ are obtained from numerical integration of $\dddot{\mathbf{q}}$ and $\dddot{\mathbf{q}}_{s}$, respectively, and $\mathbf{E}\left(t_{f}, \mathbf{s}_{k}\right)$ groups the BVP error vectors to be minimized. The parameter vector $\mathbf{s}_{k}$, used as decision variable vector to enforce the convergence of the BVP error vectors (See Algorithm 1), is defined as a vector of robot positions, velocities and accelerations, and of VSS positions, velocities and accelerations, at the time instant $t_{k}$ representing the time when the robot braking phase starts, and occurring between $t_{0}$ and $t_{f}$ of the BVP: $\mathbf{s}_{k}=\left[\mathbf{q}\left(t_{k}\right), \dot{\mathbf{q}}\left(t_{k}\right), \ddot{\mathbf{q}}\left(t_{k}\right), \mathbf{q}_{s}\left(t_{k}\right), \dot{\mathbf{q}}_{s}\left(t_{k}\right), \ddot{\mathbf{q}}_{s}\left(t_{k}\right)\right]^{T}$ as shown in Fig. 1b, $t_{k} \in\left[t_{0}, t_{f}\right]$ (only one $t_{k}$ is defined since we have a squared BVP problem). In order to define 
Table 1: RMS values of torques for nominal and VSS actuation modes.

\begin{tabular}{|c|c|c|c|c|c|c|}
\hline & & $\begin{array}{c}\text { Nominal } \\
\text { RMS. Torques }\end{array}$ & \multicolumn{2}{|c|}{$\begin{array}{c}\text { Using VSS in parallel } \\
\text { RMS. Torques }\end{array}$} & $\begin{array}{c}\text { Gain } \\
\text { Torques }\end{array}$ & $\begin{array}{c}\text { Gain } \\
\text { Energy }\end{array}$ \\
\hline Seg. & Time(s) & $\boldsymbol{\tau}_{\text {RMS }}(\mathrm{Nm})$ & $\boldsymbol{\tau}_{\text {RMS }}(\mathrm{Nm})$ & $\boldsymbol{\tau}_{v s s_{\text {RMS }}}(\mathrm{Nm})$ & $\%$ & $\%$ \\
\hline Fig. 2a & 0.51 & {$[5.65,3.41,3.85]$} & {$[0.43,0.49,0.58]$} & {$[0.48,0.56,0.59]$} & 75 & 72 \\
\hline Fig. 3a & 0.52 & {$[5.35,6.53,3.89]$} & {$[0.47,0.51,0.60]$} & {$[0.52,0.61,0.65]$} & 77 & 71 \\
\hline
\end{tabular}

the braking instant $t_{k}$, we evaluate the dynamic equations grouped in (1) for different values of $t_{k}$ on the motion segment valued from $t_{0}$ to $t_{k}$. This is done in order to choose the one which demands less input torques $\left(\boldsymbol{\tau}, \boldsymbol{\tau}_{v s s}\right)$, similar to what it is done in [15] in which the total motion segment from $t_{0}$ to $t_{f}$ is divided to solve the BVP. Finally, RK4 represents the numerical integration of the expressions in (4) by using a Runge-Kutta method, and LMUpdate is a function representing the Levenberg-Marquardt updating law based on [13].

\section{Simulation Results}

For validating the proposed approach, the numerical values of the dynamic parameters for the Delta robot were defined according to a real 3-DOF Delta robot from MG-Tech [11], with the following values: (i) proximal links (2) mass and inertia, respectively: $0.82 \mathrm{~kg}$ and $0.017 \mathrm{~kg} \cdot \mathrm{m}^{2}$ about its center of mass, located at a distance of $0.115 \mathrm{~m}$ from the rotation center of the motors $q_{i}$; (ii) parallelogram (3) mass and inertia, respectively: $0.68 \mathrm{~kg}$ and $0.024 \mathrm{~kg} \cdot \mathrm{m}^{2}$ about its center of mass, located at the middle of the parallelogram; (iii) platform mass (4): $0.72 \mathrm{~kg}$; (iv) Coulomb friction terms of the actuated joints: $0.45 \mathrm{Nm}$.

The numerical values of the dynamic parameters associated to the VSS placed in parallel to the actuated links were defined, respectively: inertias of the couplings between the motors and the VSS, $J_{1}=J_{2}=J_{3}=0.0051 \mathrm{~kg} . \mathrm{m}^{2}$, and stiffness constants, $k_{1}=k_{2}=k_{3}=4.95 \mathrm{Nm} / \mathrm{rad}$. It is worth to mention that the rotor inertias, $J_{1}, J_{2}$ and $J_{3}$ were defined according to the motor specifications in [8], whereas the spring constants of the VSS, $k_{1}, k_{2}$ and $k_{3}$ were defined according to the maximum allowable spring deformation, computed by setting the maximum input torques for the robot actuators based on the specifications in [8], and testing for several typical high-speed trajectories.

To validate the theoretical formulations, two actuation modes are considered in the numerical simulations: (i) nominal actuation, which consists of the Delta robot without springs, and with a classical fifth-degree polynomial trajectory, and (ii) by using VSS in parallel, and with the proposed formulation from Algorithm 1. Additionally, the BVP approach is tested in two scenarios according to the sequence defined in Fig. 2a and Fig. 3a, respectively. Based on the RMS values of the torques in Table 1 , it can be seen that the reduction in the full actuation chain by using VSS can reach $75 \%$ and $77 \%$ with respect to the nominal actuation mode, respectively for each task. In terms of energy reduction, from evaluating the energetic model (2), in Fig. 2b, and Fig. 3b, it can be seen that by using VSS, it is possible to reduce the energetic losses up to $71 \%$, and 


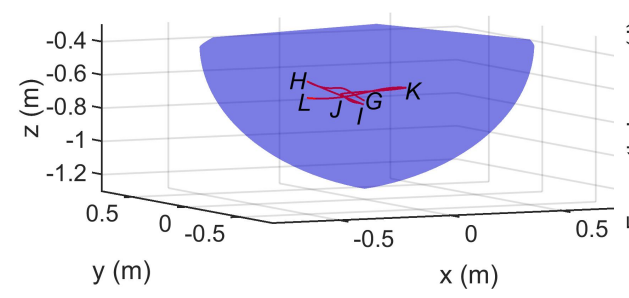

(a) Pick-and-place sequence.

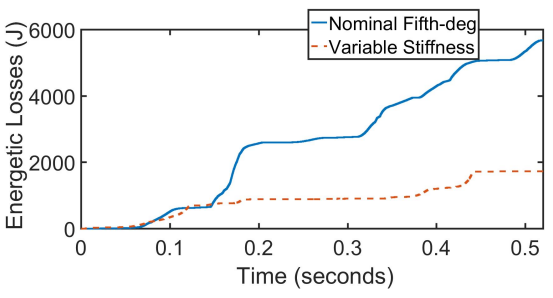

(b) Energetic losses.

Fig. 2: On the left, the sequence is defined by: $G \rightarrow H$ (time: $0.12 \mathrm{~s}$ ), $H \rightarrow I$ (time: $0.06 \mathrm{~s}$ ), $I \rightarrow J$ (time: $0.14 \mathrm{~s}$ ), $J \rightarrow K$ (time: $0.08 \mathrm{~s}$ ), $K \rightarrow L$ (time: 0.11 $\mathrm{s})$. On the right, the energetic losses for the two actuation cases.

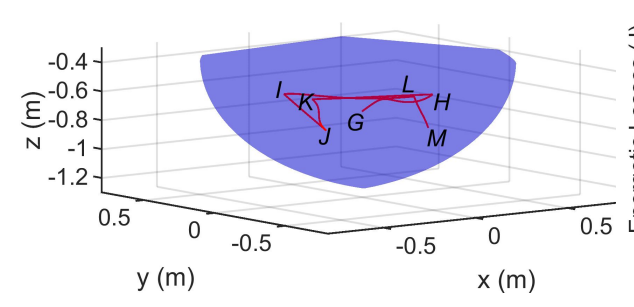

(a) Pick-and-place sequence.

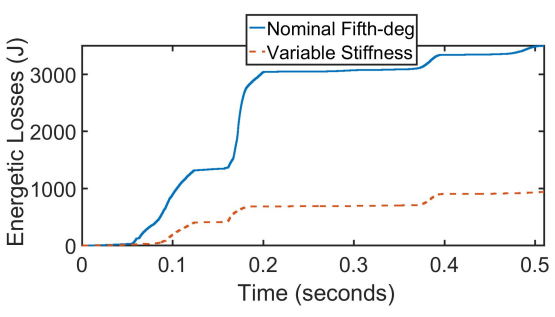

(b) Energetic losses.

Fig. 3: On the left, the sequence defined by: $G \rightarrow H$ (time: $0.12 \mathrm{~s}$ ), $H \rightarrow I$ (time: $0.06 \mathrm{~s}), I \rightarrow J$ (time: $0.1 \mathrm{~s}$ ), $J \rightarrow K$ (time: $0.06 \mathrm{~s}$ ), $K \rightarrow L$ (time: $0.1 \mathrm{~s}$ ), $L \rightarrow$ $M$ (time: $0.08 \mathrm{~s}$ ). On the right, the energetic losses for the two actuation cases.

$72 \%$, respectively, in the full actuation chain (robot and VSS) with respect to the nominal case. It is worth noticing that here only two scenarios in Fig. 2a and Fig. 3a are presented. Nevertheless, other simulations, not presented here due to page limitations, gave similar results in terms of energy reduction.

\section{Conclusions and future works}

This paper proposes a strategy for generating offline optimal motions in order to increase the energy efficiency of a Delta robot by placing variable stiffness springs (VSS) in parallel to the robot active joints. The VSS in parallel configuration was combined with a BVP to find a combined optimal motion of robot and VSS joints. Simulations led to a considerably increase of energy efficiency with multiple-point trajectories on a Delta robot for two cases: nominal (without VSS) and by using VSS. Results show that by using VSS, the energy reduction for the full actuation chain on the Delta robot, i.e. robot-plus-VSS, can reach up to $70 \%$ with respect to the nominal case. The future works include the development of a strategy that takes into account the uncertainties on the dynamic parameters as here we assume the numerical integration of a perfect model. In addition to that, future works include as well the experimental validation of our approach.

\section{Acknowledgment}

This work was conducted with the support of the Mexican Council for Science and Technology (CONACYT), which had fund a PhD grant. 


\section{Bibliography}

[1] M. Brossog, M. Bornschlegl, J. Franke: Reducing the energy consumption of industrial robots in manufacturing systems. International Journal of Advanced Manufacturing Technology. May 2015, Vol. 78 Issue 5-8, pp. 1315-1328.

[2] Y. J. Kim, 2017. Anthropomorphic Low-Inertia High-Stiffness Manipulator for High-Speed Safe Interaction. In IEEE Transactions on Robotics, vol. 33, No. 6, pp. 1358-1374, December 2017.

[3] S. Briot, and A. Goldsztejn, 2018. Topology Optimization of Industrial Robots: Application to a Five-bar Mechanism. In Mechanism and Machine Theory, 2018, Vol. 120, pp. 30-56.

[4] M. Uemura and S. Kawamura: Resonance-based motion control method for multijoint robot through combining stiffness adaptation and iterative learning control. In IEEE International Conference on Robotics and Automation, Kobe, Japan, pp. 1543-1548, 2009.

[5] J.P. Barreto, F.J.-F. Schöler and B. Corves: The concept of natural motion for pick and place operations. In: New advances in mechanisms, mechanical transmissions and robotics. Springer, pp. 89-98.

[6] J.P. Barreto, and B. Corves: Resonant Delta Robot for Pick-and-Place Operations, In Advances in Mechanism and Machine Science, pp. 2309-2318, 2019.

[7] H. Goya, K. Matsusaka, M. Uemura, Y. Nishioka, and S. Kawamura: Realization of high-energy efficient pick-and-place tasks of scara robots by resonance. In IEEE/RSJ International Conference on Intelligent Robots and Systems (IROS 2012), pp. 2730-2735, October 2012, Vilamoura, Algarve (Portugal).

[8] R. Balderas Hill, S. Briot, A. Chriette, and P. Martinet, 2018. Increasing energy efficiency of high-speed parallel robots by using variable stiffness springs and optimal motion generation. Proceedings of the ASME 2018 IDETC/CIE 2018, pp. V05BT07A011 10 pages, DETC2018-85090, Quebec City, Canada.

[9] R. Balderas Hill, S. Briot, A. Chriette, and P. Martinet, 2018. Minimizing Input Torques of a High-Speed Five-Bar Mechanism by using Variable Stiffness Springs. Proceedings of the 22nd CISM-IFToMM Symposium on Robot Design, Dynamics, and Control (ROMANSY 2018), pp. 61-68, June 25-28, 2018, Rennes, France.

[10] K. Hollander and T. Sugar. Concepts for compliant actuation in wearable robotic systems. In Proc. of the US-Korea Conference on Science, Technology and Entrepreneurship (UKC04), 2004, vol. 128, pp. 644-650.

[11] MG-Tech. URL https://www.mg-tech.fr/

[12] S. Briot and W. Khalil: Dynamics of Parallel Robots: From Rigid Bodies to Flexible Elements. Springer, 2015. ISBN 9783319197883.

[13] J.J. Mor, 1978. The Levenberg-Marquardt algorithm: Implementation and theory. In: Watson G.A. (eds) Numerical Analysis. Lecture Notes in Mathematics, vol 630. Springer, Berlin, Heidelberg.

[14] C. Gosselin, and J. Angeles. 1990. Singularity analysis of closed-loop kinematic chains. IEEE Trans. on Rob. and Aut., 6(3), pp. 281-290.

[15] R. Balderas Hill, S. Briot, A. Chriette and P. Martinet, 2019. Exploiting Natural Dynamics in order to Increase the Feasible Static-Wrench Workspace of Robots. Proceedings of the 15th IFToMM World Congress, June 30July 4, 2019, Krakow, Poland. 